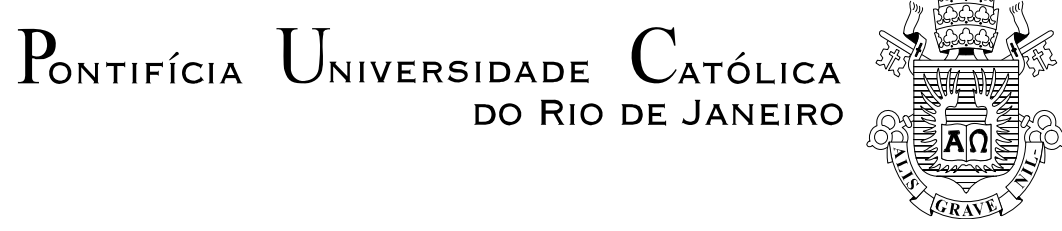

Sylvia Telles Ribeiro

\title{
Precificação Ótima dos Contratos de Gás Natural na Modalidade Interruptível
}

\author{
Dissertação de Mestrado
}

Dissertação apresentada como requisito parcial para obtenção do grau de Mestre pelo Programa de Pósgraduação em Engenharia Elétrica do Departamento de Engenharia Elétrica da PUC-Rio.

Orientador: Prof. Alexandre Street de Aguiar

Co-orientador: Prof. Cristiano Augusto Coelho Fernandes

Rio de Janeiro

Abril de 2009 


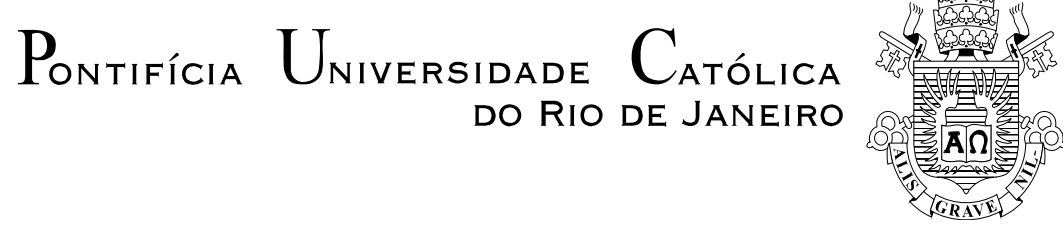

Sylvia Telles Ribeiro

\title{
Precificação Ótima dos Contratos de Gás Natural na Modalidade Interruptível
}

Dissertação apresentada como requisito parcial para obtenção do grau de Mestre pelo Programa de Pós-Graduação em Engenharia Elétrica do Departamento de Engenharia Elétrica do Centro Técnico Científico da PUC-Rio. Aprovada pela Comissão Examinadora abaixo assinada.

\author{
Dr. Alexandre Street de Aguiar \\ Orientador \\ Departamento de Engenharia Elétrica - PUC-Rio \\ Dr. Cristiano Augusto Coelho Fernandes \\ Co-Orientador \\ Departamento de Engenharia Elétrica - PUC-Rio
}

Dr. Luiz Augusto Nóbrega Barroso

PSR Consultoria

Dr. Delberis Araújo Lima

Departamento de Engenharia Elétrica - PUC-Rio

Prof. José Eugenio Leal Coordenador Setorial do Centro

Técnico Científico

Rio de Janeiro, 28 de abril de 2009 
Todos os direitos reservados. É proibida a reprodução total ou parcial do trabalho sem autorização da universidade, da autora e do orientador.

\section{Sylvia Telles Ribeiro}

Graduou-se em Economia na PUC-RJ em 2003. Desde 2004, trabalha com gestão e otimização de Portfólio da área de Gás e Energia da Petrobras.

Ficha Catalográfica

Ribeiro, Sylvia Telles

Precificação Ótima dos Contratos de Gás Natural na Modalidade Interruptível / Sylvia Telles Ribeiro; orientador: Alexandre Street de Aguiar; co-orientador: Cristiano Augusto Coelho Fernandes. - 2009.

78 f. ; $30 \mathrm{~cm}$

Dissertação (Mestrado em Engenharia Elétrica)Pontifícia Universidade Católica do Rio de Janeiro, Rio de Janeiro, 2009.

Inclui bibliografia

1. Engenharia elétrica - Teses. 2. Precificação ótima. 3. Contratos Interruptíveis. 4. Otimização Estocástica 5 - Medidas de Risco. I. Aguiar, Alexandre Street de, II. Fernandes, Cristiano Augusto Coelho. III. Pontifícia Universidade Católica do Rio de Janeiro. Departamento de Enqenharia Elétrica. IV. Título. 
À minha mãe, Marina Figueira de Mello. Por tudo. 


\section{Agradecimentos}

À Minha família, que me deu todo amor, proteção e suporte que precisei nessa vida, com especial carinho para meu irmão Guilherme, meu tio Sérgio, meu tio Gustavo, e Amilcar.

Às minhas amoras, que sempre me apoiaram em todos os momentos importantes da minha vida. Dentre elas, destaco Amanda Cavalcanti, Andrea Canedo, Luisa Prochnik, Izabel Ramos, Joana Cotrim, Julia Boohrem, Márcia Seixas, Patrícia Fernandes e Raquel Tessarollo.

Aos meus expoentes profissionais, estrelas que brilharam para mim e me ajudaram a brilhar profissionalmente: Mario Jorge, Alexandre Silvestre, , João Alberto, Marcus Moacyr, Luciana Rachid e Graça Foster.

Aos meus colegas de equipe da PAE, por todo apoio e compreensão durante o desenvolvimento deste trabalho.

Aos meus queridos professores, que me abriram tantas portas e acreditaram em mim, Rogério Werneck, Cristiano Fernandes e Marco Antônio Guimarães.

Um agradecimento especial ao meu orientador Alexandre Street, por ter sido incansável, um apoio mais que indispensável à realização desse trabalho. 


\section{Resumo}

Ribeiro, Sylvia Telles; Aguiar, Alexandre Street de (Orientador); Fernandes, Cristiano Augusto Coelho (Co-Orientador). Precificação Ótima dos Contratos de Gás Natural na Modalidade Interruptível. Rio de Janeiro, 2009. 78p. Dissertação de Mestrado - Departamento de Engenharia Elétrica, Pontifícia Universidade Católica do Rio de Janeiro.

O segmento industrial desempenha um importante papel no desenvolvimento do setor de gás Brasileiro. Em função dos baixos preços e dos incentivos dados pelo governo para a conversão dos processos industriais (muitos deles dependentes do óleo combustível) para o gás natural, criou-se uma fonte de demanda "firme" deste combustível. Como as termelétricas operam em regime de complementariedade ao sistema hidrelétrico (sendo coordenadas pelo Operador Nacional do Sistema (ONS) elétrico e chamadas a gerar apenas em situações hidrológicas "desfavoráveis"), o oconsumo de gás termelétrico ocorre de forma esporádica. Uma forma de se aumentar a eficiência do uso do gás, mesclando duas classes de consumidores se dá através dos contratos interruptíveis, que proporcionam ao produtor a capacidade de atender consumidores industriais bicombustível (gás e óleo por exemplo) com o gás ocioso das termelétricas. Como a atratividade deste contrato depende do desconto dado com relação ao preço do contrato firme, que não é interrompido, o objetivo deste trabalho é a construção de um modelo analítico para a determinação do preço ótimo dos contratos de fornecimento de gás interruptíveis, por parte de um produtor monopolista. O consumo de gás das termelétricas será considerado como principal fonte de incerteza do modelo, que por sua vez será caracterizada através de cenários de operação ótima do sistema elétrico, simulados conforme a metodologia utilizada pelo ONS. O perfil de risco do produtor será caracterizado pelo Conditional Value-at-Risk (CVaR).

\section{Palavras-chave}

Gás Natural, Termelétrica, GNL, Contratos de Fornecimento Interruptível, CVaR, Otimização Estocástica, Decisão sob Incerteza, risco. 


\section{Abstract}

Ribeiro, Sylvia Telles; Aguiar, Alexandre Street de (Advisor); Fernandes, Cristiano Augusto Coelho (Co-Advisor). Optimal Pricing of Natural Gas Flexible Contracts. Rio de Janeiro, 2009. 78p. MSc Dissertation Departamento de Engenharia Elétrica, Pontifícia Universidade Católica do Rio de Janeiro.

Brazilian natural gas industry growth has been led by electricity supply. As hydro plants generate at lower costs, thermal units only produce when hydro electricity is insufficient. This makes natural gas consumption highly volatile: Either all thermal units generate together or don't. When all units generate together, the gas trader has to buy LNG - Liquified Natural Gas at the spot market incurring price risk. This risk can be mitigated in case the gas trader is able to sell flexible contracts to the industrial sector that can be interrupted in case of thermal generation. Thus the gas volume sold under flexible contracts is used either by thermal generation or by the industrial sector, virtually reducing total demand and avoiding emergency LNG purchases. The determination of the optimal price for these contracts is the aim of this dissertation. The determination model proposed will try to maximize a convex combination of CVaR - Conditional Value at Risk NPV - Net Present Value and trader's profit NPV.

\section{Keywords}

Natural gas, Thermal plants, Flexible Contracts, CVaR, Stocastic Optimization, Decision under Uncertainty, Risk. 


\section{Sumário}

1 Introdução 13

1.1 Histórico e Motivação 13

1.2 Lucro do Comercializador no fornecimento de gás natural 20

1.3 Objetivo 22

$\begin{array}{lll}1.4 & \text { Organização da Dissertação } & 23\end{array}$

2 Mercado de Gás Natural no Brasil 25

2.1 Consumo de gás - termelétrico vs não termelétrico 25

2.2 Contratos Firmes 28

2.3 Contratos Preferenciais ou Termelétricos 29

2.4 Contratos Interruptíveis 30

3 Medidas de Risco e Decisão sobre incerteza 34

3.1 Decisão Sob Incerteza 34

3.2 Modelagem das Incertezas $\quad 35$

3.3 Teoria da Utilidade 36

3.4 Medidas de Risco 36

$\begin{array}{lll}3.4 .1 & \text { Teoria de Portfólio de Markowitz } & 37\end{array}$

3.4.2 O VaR - Value at Risk 39

3.4.3 O CVaR - Conditional Value at Risk 40

4 Metodologia para Determinação do Preço Ótimo para Contratos Interruptíveis 43

4.1 Oferta e Demanda de Gás Natural no Brasil. 43

4.1.1 Composição da Oferta 43

4.1.2 Balanço de Compra e Venda 45

4.2 Equação de Lucro do Comercializador 46

4.2.1 Formação dos Custos $\quad 46$

4.2.2 Formação das Receitas $\quad 50$

4.3 O Problema de otimização do Comercializador 51

5 Estudo de caso 55

5.1 Premissas de Receitas e Custos $\quad 55$

5.1.1 Preços Firme e Termoelétrico $\quad 55$

$\begin{array}{lll}5.1 .2 & \text { Preços de GNL } & 56\end{array}$

5.1.3 Custo do Gás Firme 58

5.1.4 Quantidades Contratadas $\quad 59$

5.2 Premissas de Despacho 60

$\begin{array}{lll}5.3 \text { Penalidades } & 60\end{array}$

$\begin{array}{ll}5.4 \text { Horizonte Temporal } & 61\end{array}$

5.5 Outras Premissas Relevantes 62

$\begin{array}{lll}5.6 & \text { Resultados } & 62\end{array}$

5.7 Equilíbrio Competitivo versus Equilíbrio de Monopólio 64

$\begin{array}{ll}5.8 \text { Sensibilidades } & 67\end{array}$

5.8.1 Sensibilidades em relação à $\lambda$ : $\quad 67$

5.8.2 Sensibilidades para Preço de GNL $\quad 69$ 
5.8.3 Outras Sensibilidades de Interesse $\quad 70$

6 Conclusões e Trabalhos Futuros $\quad 72$

6.1 Trabalhos Futuros $\quad 73$

7 Referências bibliográficas $\quad 74$

ANEXO A - Cálculo da Curva de Disposição a Contratar dos

Consumidores Industriais 


\section{Lista de Figuras}

Figura 1.1 - Histórico e Motivação....................................................... 13

Figura 1.2 - Evolução da Matriz Energética Brasileira......................... 16

Figura 1.3 - Balanço de Oferta e Demanda de Gás Natural no Brasil... 17

Figura 2.1 - Sazonalidade no Consumo de Gás Natural Argentino...... 26

Figura 2.1 - Tornando firme um consumo flexível............................. 32

Figura 2-2 - Atendimento dos Contratos Flexíveis............................ 32

Figura 3.1 - Decisões de Dois Estágios................................................ 35

Figura 3.2 - Fronteira Eficiente de Markowitz.................................... 38

Figura $3.3-\mathrm{VaR}-$ Value at Risk.............................. 39

Figura 3.4 - CVaR - Conditional Value at Risk.................... 41

Figura 4.1 - Balanço de Compra e Venda............................................ 45

Figura 4.2 - Prêmio do GNL como Função do volume Demandado.... 49

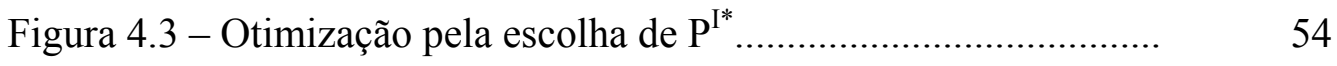

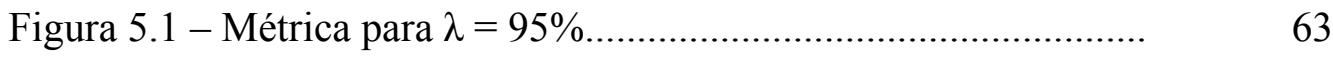

Figura 5.2 - Equilíbrio Competitivo vs Monopolista........................... 66

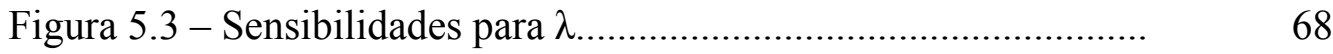

Figura 5.4 - Sensibilidades para Preços de GNL.................................. 69

Figura A.1 - Distribuição do consumo industrial de gás para geração de energia. 


\section{Lista de Tabelas}

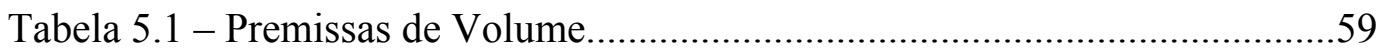

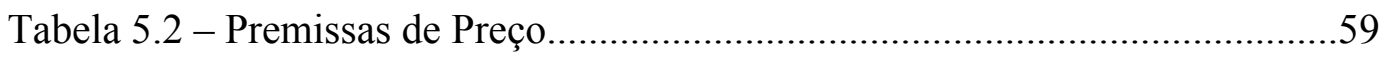

Tabela 5.3 - Comparação entre hipótese competitiva e modelo monopolista

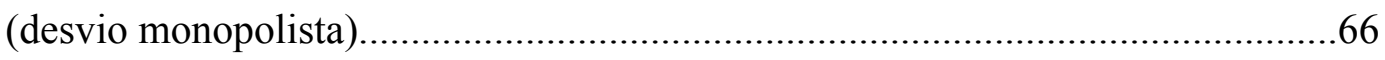

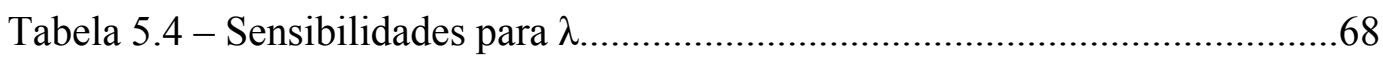

Tabela A.1 - Curva de Demanda por contratos interruptíveis como função do

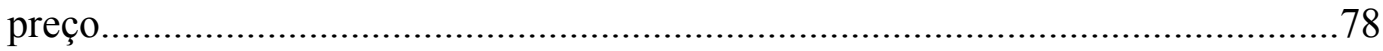




\title{
Siglas utilizadas
}

\author{
GNL - Gás Natural Liquefeito \\ SDDP - stochastic dual dynamic programming \\ VaR - Value at Risk \\ cVaR - Conditional Value at Risk.Introdução
}

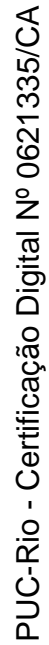

